\title{
Condición Periodontal de las Prótesis Fijas Singulares Realizadas en la Clínica Odontológica Integral del Adulto de la Universidad Mayor de Temuco
}

\author{
Periodiontal Condition of Fixed Single Prostheses Made at Integral \\ Adult Dental Clinic of the Universidad Mayor in Temuco
}

Luis Bustos Medina* \& Carla Oyanader Martínez**

BUSTOS, M. L. \& OYANADER, M. C. Condición periodontal de las prótesis fijas singulares realizadas en la clínica odontológica integral del adulto de la universidad mayor de Temuco. Int. J. Odontostomat., 6(2):195-200, 2012.

RESUMEN: El objetivo de esta investigación fue determinar la condición periodontal en prótesis fijas singulares (PFS) realizadas en la clínica odontológica Integral del Adulto I y II de la Universidad Mayor de Temuco entre los años 2007 y 2009 . Se evaluaron 73 PFS mediante un examen clínico, realizadas en 41 pacientes. Se analizaron las variables: edad, sexo, pieza dentaria, año de atención, año académico en que se realizaron las PFS, material de las prótesis fijas; los parámetros periodontales evaluados en este estudio fueron edema y eritema, sangramiento y recesión gingival. La frecuencia de edema y eritema fue de $81 \%$, el sangramiento lo encontramos en un $77 \%$ y la recesión gingival en un $27 \%$. Las mujeres presentaron mayores problemas de los tejidos periodontales con un $85 \%$ de edema y eritema y un $81 \%$ de sangramiento. Las PFS realizadas en el 4 to año académico presentaron un $55 \%$ de recesión gingival y las realizadas en el 5to año mostraron un $17 \%$. En relación al año de atención en el que se realizaron las rehabilitaciones, las PFS realizadas en el año 2007 presentaron un 100\% de inflamación, mientras que en los otros años (2008 y 2009) las PFS presentaron menos daño a nivel gingival. Las piezas más afectadas según la ubicación en la boca, fueron las posteriores con un $84 \%$ de edema y eritema. Respecto al material, las PFS que presentaron más problemas de edema y eritema fueron las de metal cerámica con un $82 \%$. Del total de los 41 pacientes observados, la mayoría estaban satisfechos con su rehabilitación. Finalmente se puede concluir que no existió evidencia estadísticamente significativa para concluir que existe una asociación entre las variables edad, sexo, año de atención y las variables edema y eritema, sangramiento y recesión gingival. En cambio, si se encontró evidencia estadísticamente significativa entre el cuarto y quinto año académico/ durante el tiempo/ durante los años académicos en el que se realizaron las PFS y la recesión gingival.

PALABRAS CLAVE: restauraciones, salud periodontal y gingival, de cerámica, complicaciones biológicas, de metal-cerámica, coronas individuales.

\section{INTRODUCCIÓN}

Se ha demostrado que las restauraciones fijas provocan cambios en los tejidos periodontales en mayor y menor medida, durante todos los procedimientos que se llevan a cavo para su realización (Castellani, 1996).

Con el fin de conseguir resultados estéticos y funcionales a largo plazo, el clínico debe asegurarse de que el tejido gingival esté sano antes de comenzar con la preparación definitiva del diente, al igual que en el momento de cementar la prótesis fija singular (PFS) (Donovan \& Cho, 2002; Padbury et al., 2003), ya que un periodonto saludable reaccionará en forma predecible y positiva a la agresión inevitable que producen nuestras intervenciones, en una situación de patología esta agresión empeorará las condiciones del tejido (Castellani; Newman et al., 2010). Es por esto, que es importante que la gingivitis y periodontitis sean tratadas satisfactoriamente y eliminadas previas a comenzar el tratamiento, y esta condición de salud debe permanecer durante todo el procedimiento; por lo cual la meta de una rehabilitación protésica y periodontal, debe ser que permita un control óptimo de la placa dental.

\footnotetext{
* Centro de Excelencia CIGES (Capacitación, Investigación y Gestión para la Salud Basada en la Evidencia). Dpto. Salud Pública. Facultad de Medicina, Universidad de La Frontera, Temuco, Chile.

** Cirujano Dentista, Universidad Mayor, Temuco, Chile.
} 
Además se debe instalar citas de mantención a intervalos que dependan de las condiciones particulares del paciente.

El tejido dentario perdido debe ser sustituido con un material que sea biocompatible con los tejidos gingivales, que no permita la formación de placa y que no contribuya a la inflamación gingival. Mediante restauraciones de metal cerámica y libre de metal se pueden reemplazar grandes áreas de estructura dentaria perdidas, al mismo tiempo que protege y preserva el remanente dentario (Shillingburg et al., 2000).

El objetivo de este estudio fue evaluar la condición periodontal de las prótesis fijas singulares realizadas en la clínica Odontológica Integral del Adulto I y II de la Universidad Mayor de Temuco entre los años 2007 y 2009.

\section{MATERIAL Y MÉTODO}

Estudio de corte transversal. Se seleccionó una muestra no probabilística de 73 prótesis fijas singulares. El criterio de inclusión fue pacientes atendidos en la clínica odontológica de la Universidad Mayor de Temuco en las asignaturas Odontología Integral del Adulto I y II que se realizaron prótesis fijas singulares (PFS) en el periodo 2007 al 2009. El criterio de exclusión fue, pacientes que se realizaron PFS metálicas y de resina.

Se realizó el examen y evaluación, por una estudiante de sexto año de la carrera de odontología, este fue realizado en el horario de atención de la clínica odontológica de diagnóstico, entre el 7 de abril y 20 de abril del año 2010.

Se inició con las preguntas de los datos demográficos y anamnesis relacionada con su tratamiento en general y luego en relación a la pieza a evaluar. A continuación, se hizo el examen clínico intraoral, consistente en pesquisar alguna anomalía en relación a la pieza rehabilitada, además de ver la ubicación de ésta en el arco dental y material de la PFS. Posteriormente se realizó el examen periodontal; edema, eritema, sangramiento y se realizaron mediciones con sonda periodontal CP-11 HU-FRIEDY de profundidad al sondaje y nivel de inserción clínica. Por último se consultó a los pacientes si estaban o no satisfechos con la rehabilitación.
Durante el desarrollo de las evaluaciones, se asignó un número a cada ficha, de esta forma se facilitó el ingreso a la base de datos y posterior análisis. Para realizar el ingreso de datos se utilizó el programa Microsoft Office Excel 2007 y para efectuar el análisis el programa Stata 11.0

Se realizó un análisis descriptivo a través de tablas estadísticas, con frecuencia, promedio y desviación estándar. La comparación de los porcentajes fue con la prueba exacta de Fisher y el nivel de significación fue $5 \%$.

\section{RESULTADOS}

Se examinaron 73 prótesis fijas singulares realizadas por alumnos de la asignatura Odontología Integral del Adulto I y II de la Universidad Mayor de Temuco, en el periodo 2007 al 2009. La distribución de la muestra por edad y sexo, indica que de 41 pacientes, $76 \%$ eran mujeres. La edad de los pacientes fluctuaba entre los 23 y 66 años, con un promedio de edad de 45,5 $\pm 9,8$ años.

Del total de las 196 PFS realizadas se pudieron evaluar $73 ; 7 \%$ del año $2007,52 \%$ del 2008 y $52 \%$ del año 2009. Se evaluó también la ubicación de las PFS en la boca, el material con el que fueron realizadas y la frecuencia de alteración periodontal (Tabla I).

Del total de las restauraciones evaluadas, tanto el eritema, edema y sangramiento fueron más frecuentes en mujeres, mientras que la recesión gingival lo fue en hombres, pero estas diferencias no fueron estadísticamente significativas. Las PFS realizadas en 4to año, presentan más alteraciones de los tejidos gingivales que las realizadas en 5to; estas diferencias fueron estadísticamente significativas para asociar curso con recesión gingival, pero no con edema, eritema y sangramiento.

En relación al año en que se realizaron las rehabilitaciones, las PFS realizadas el año 2007 presentan un $100 \%$ de las edema, eritema y sangramiento y un $40 \%$ de recesión gingival, mientras que los otros años las PFS presentan menos daño a nivel gingival. Según la posición en la boca, las piezas más afectadas por alteraciones gingivales fueron las posteriores. Respecto al material, las PFS que presentan más problemas de edema y eritema eran las de metal-cerámica, respecto al sangramiento no se encontró diferencia, ya que 
los porcentajes fueron similares. Al evaluar la presencia de recesión las PFS que más la presentaron fueron las de cerámica. No existió evidencia estadísticamente sig- nificativa para concluir que exista asociación entre año atendido, ubicación de la PFS en la boca y material de esta con alteración gingival (Tabla II).

Tabla I. Distribución de las PFS según ubicación en la boca, material y alteración periodontal.

\begin{tabular}{lllll}
\hline Parámetro de PFP & & $\mathbf{n}$ & \% \\
\hline \multirow{2}{*}{ Ubicación del diente en la arcada } & Anterior & & 42 & 58 \\
& Posterior & 31 & 42 \\
& Metal cerámica & & 60 & 82 \\
& Cerámica & & 13 & 18 \\
& & Si & 59 & 81 \\
Alteración periodontal & Edema y Eritema & No & 14 & 19 \\
& & Si & 56 & 77 \\
& Sangramiento & No & 17 & 23 \\
& & Si & 20 & 27 \\
& Recesión gingival & No & 53 & 73 \\
\hline
\end{tabular}

Tabla II. Asociación entre sexo, curso, año en que se realizó la rehabilitación, ubicación de la pieza en el arco dental, material de la PFS y presencia se alteración gingival. Prueba exacta de Fisher.

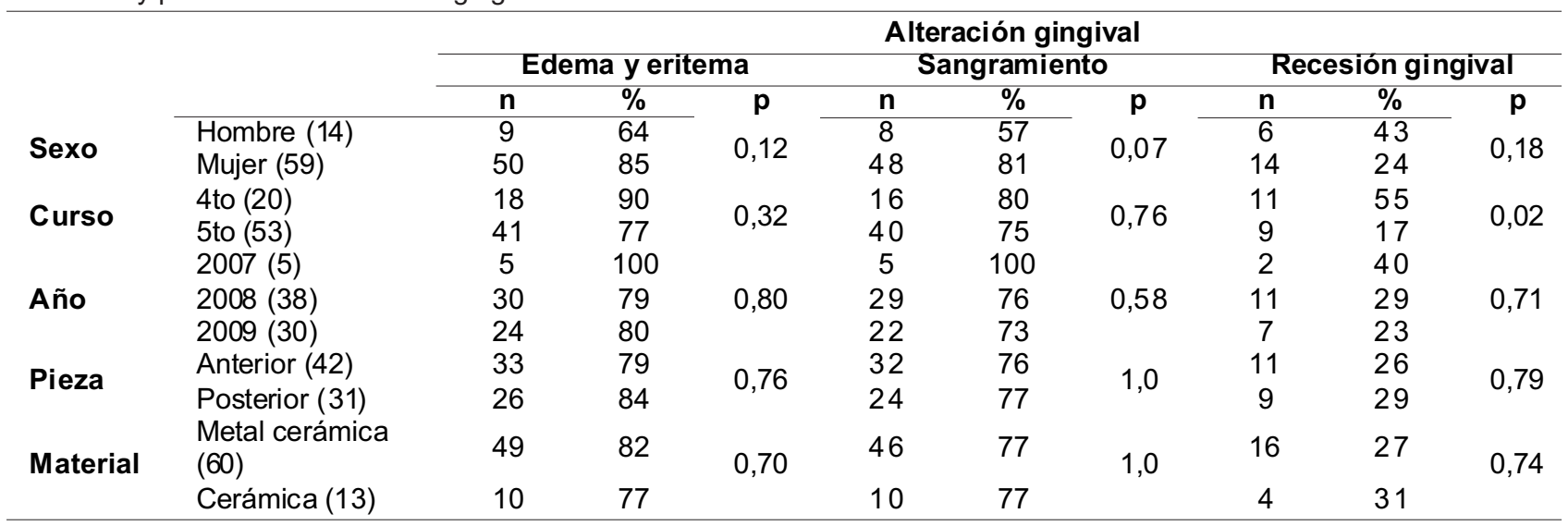

Tabla III. Grado de satisfacción de los pacientes atendidos en la clínica odontológica de la Universidad Mayor. Prueba exacta de Fisher.

\begin{tabular}{|c|c|c|c|c|c|c|}
\hline & & \multicolumn{5}{|c|}{ Grado de satisfacción } \\
\hline & & \multicolumn{2}{|c|}{ Satisfecho } & \multicolumn{3}{|c|}{ No satisfecho } \\
\hline & & $\mathbf{n}$ & $\%$ & $\mathbf{n}$ & $\%$ & $\mathbf{p}$ \\
\hline \multirow{2}{*}{ Sexo } & Hombre & 12 & 86 & 2 & 14 & \multirow{2}{*}{1,0} \\
\hline & Mujer & 48 & 81 & 11 & 19 & \\
\hline \multirow{3}{*}{ Curso } & 4 to & 19 & 95 & 1 & 5 & \multirow{3}{*}{0,09} \\
\hline & 5 to & 41 & 77 & 12 & 23 & \\
\hline & 2007 & 5 & 100 & 0 & 0 & \\
\hline \multirow[t]{2}{*}{ Año atendido } & 2008 & 32 & 84 & 6 & 16 & \multirow[t]{2}{*}{0,55} \\
\hline & 2009 & 23 & 77 & 7 & 23 & \\
\hline \multirow{2}{*}{ Pieza } & Anterior & 35 & 83 & 7 & 17 & \multirow{2}{*}{0,76} \\
\hline & Posterior & 25 & 81 & 6 & 19 & \\
\hline \multirow{2}{*}{ Material } & Metal cerámica & 48 & 80 & 12 & 20 & \multirow{2}{*}{0,44} \\
\hline & Cerámica & 12 & 92 & 1 & 8 & \\
\hline
\end{tabular}


Tabla IV. Asociación del promedio de edad con presencia de alteraciones gingivales. Prueba t-test para varianzas iguales.

\begin{tabular}{llcccc}
\hline & & \multicolumn{2}{c}{ Edad } & & \\
\cline { 3 - 6 } & & Promedio & DE & n & p \\
\hline \multirow{2}{*}{ Eritema y edema } & $\mathrm{Si}$ & 45,3 & 9,9 & 59 & 0,72 \\
& $\mathrm{No}$ & 46,4 & 9,3 & 14 & \\
\multirow{2}{*}{ Sangramiento } & $\mathrm{Si}$ & 45,5 & 9,9 & 56 & \multirow{2}{*}{0,98} \\
& $\mathrm{No}$ & 45,5 & 9,6 & 17 & \\
\multirow{2}{*}{ Recesión gingival } & $\mathrm{Si}$ & 43,9 & 10,3 & 20 & \multirow{2}{*}{0,37} \\
& $\mathrm{No}$ & 46,2 & 9,6 & 53 & \\
\hline
\end{tabular}

Del total de los 41 pacientes atendidos, la mayoría estaban satisfechos con su rehabilitación, sin embargo, las mujeres fueron las que presentaron menos aceptación de ésta. Los pacientes que se atendieron con alumnos de 4to año, se encontraban más complacidos con el resultado de sus PFS, siendo el año 2007 en que no presentó ninguna queja al respecto. Los pacientes que rehabilitaron sus dientes anteriores y con restauraciones de cerámica libre de metal eran los más satisfechos de sus rehabilitaciones. No existió evidencia estadísticamente significativa para concluir asociación entre sexo, curso atendido, año de atención, pieza y material de la PFS y satisfacción del paciente (Tabla III).

La distribución de la edad fue similar en todas las variables que midieron alteraciones gingivales, con un promedio mínimo de 44 y máximo de 46 años (Tabla IV).

\section{DISCUSIÓN}

De las 73 rehabilitaciones realizadas mediante prótesis fijas singulares, se observa que el $81 \%$ del tejido gingival circundante de las PFS presentaron edema y eritema; comparados con los resultados obtenidos por Arango et al. (1995) donde un 90,7\% presentó inflamación. El sangramiento al sondaje en nuestro estudio fue $77 \%$ en las piezas evaluadas, en los realizados por Näpänkanga et al. (1997) y Näpänkanga \& Raustia (2008), el sangrado de las encías alrededor de las PFS fue menor, con un $27 \%$ y $25 \%$ de los sujetos del estudio.

Con respecto al material de las PFS; concordando con la revisión realizada por Sailer et al. (2007), las PFS que tienes más alteraciones a nivel gingival son las con material tipo metal-cerámica. En nuestro estudio un $77 \%$ de las PFS de cerámica pura presentaron alteraciones de los tejidos periodontales, estos resultados son mucho mayores a los encontrados por Goodacre et al. (2003), quién de un total de 4277 PFS evaluadas, un $8 \%$ presentaron algún tipo de complicación.

Newman et al. encontraron que es más difícil predecir con precisión el pronósticos para los dientes posteriores que para los dientes anteriores. Esta situación fue observada en nuestro estudio ya que las piezas más afectadas fueron las posteriores, pues presentaron edema y eritema en un $84 \%$ en comparación al $79 \%$ de las piezas anteriores.

Con respecto al año de realización de las PFS, las realizadas en 4 to año presentaron un $90 \%$ de edema y eritema, $80 \%$ de sangramiento y $55 \%$ recesión, en comparación a los porcentajes obtenidos en las rehabilitaciones de 5to año que fueron menores; lo que se puede deber a la menos experiencia y tienen menos incorporado el manejo adecuado de los tejidos blandos de los alumnos de OIA I ya que es su primer año clínico.

Del total de las restauraciones evaluadas todos los signos de inflamación fueron de mayor frecuencia en las pacientes mujeres: un $85 \%$ de edema y eritema y $81 \%$ de sangramiento. En este sentido podríamos mencionar que durante los periodos de fluctuación hormonal se han identificado modificaciones clínicas en los tejidos periodontales; Mealey \& Moritz (2003) y Güncü et al. (2005) en sus respectivas revisiones determinaron que las variaciones hormonales que experimentan las mujeres en situaciones fisiológicas (pubertad, menstruación, embarazo y menopausia) y no fisiológicas (anticonceptivos orales) producen cambios significativos en el periodonto. 
Bader et al. (1991), mostró en su estudio realizado entre 1987 y 1989 que la recesión gingival en los dientes que recibieron y en los dientes que no recibieron PFS, no fueron estadísticamente significativos; además de este estudio no se encontró en la literatura relación entre recesiones y piezas rehabilitadas con PFS, los únicos estudios revisados, fueron evaluaciones realizadas en piezas dentales.

En un estudio realizado por Linares (2008), se halló una recesión del $85 \%$ en algún grado de acuerdo a la clasificación de Miller, en nuestro estudio se observó una recesión de $27 \%$ según la clasificación de Miller. Maetahara (2006), registró mayores porcentajes de recesión gingival según sexo en los pacientes hombres con $84,5 \%$. En nuestro estudio registramos en similares condiciones un $43 \%$ de recesión gingival, afectando un $29 \%$ de las piezas posteriores y $26 \%$ de las piezas anteriores. En este sentido Maetahara registró que las piezas dentarias más afectadas fueron los premolares con $27,5 \%$ y las piezas dentarias con menor afectación fueron los caninos con 14,6\%. Dautovic-Kazazaic et al. (2010), en un estudio sobre PFS metal cerámica encontró retracción gingival en un $62,5 \%$. En nuestro estudio encontramos un $27 \%$ de recesión.

Respecto al grado de satisfacción de los pacientes con sus PFS, Näpänkangas et al. y Näpänkangas \& Raustia, describieron en sus estudios que los pacientes se mostraron satisfechos tanto en estética, color, forma y acabado de las PFS, $93 \%$ y $97 \%$ respectivamente. Estos porcentajes son un poco mayor a los encontrados en nuestro estudio que fue un $82 \%$.

BUSTOS, M. L. \& OYANADER, M. C. Periodiontal condition of fixed single prostheses made at integral adult dental clinic of the Universidad Mayor in Temuco. Int. J. Odontostomat., 6(2):195-200, 2012.

ABSTRACT: The aim of this study was to determine the periodontal condition in fixed single prostheses made at Integral Adult Dental Clinic I and II of the Universidad Mayor in Temuco, between the years 2007 and 2009. Seventy-three fixed single prostheses were evaluated by clinical examination, made in 41 patients. Variables were analyzed: age, sex, tooth, year of care, year in which the fixed single prostheses were made, fixed single prostheses material; periodontal parameters evaluated in this study were edema and erythema, bleeding and gingival recession. Edema and erythema's frequency were $81 \%$, bleeding in $77 \%$ and gingival recession in $27 \%$. Women presented major problems in periodontal tissues with $85 \%$ of edema and erythema and $81 \%$ of bleeding. Fixed single prostheses made in 4 th year showed $55 \%$ of gingival recession and the ones that were made in 5th year showed $17 \%$. In relation to the year of care in which the rehabilitations were made, the fixed single prostheses made in 2007 showed $100 \%$ of inflammation, while in the other years (2008 and 2009) the fixed single prostheses showed less damage at gingival level. The most affected teeth according to the location in the mouth were the back ones with $84 \%$ of edema and erythema. Regarding material, the fixed single prostheses that showed more edema and erythema problems were the metal-ceramics with $82 \%$. Of the total of the 41 patients observed, the majority was satisfied with their rehabilitation. Finally we can conclude that there was no statistically significant evidence to conclude that there is an association between the variables age, sex, year of care and the variables edema and erythema, bleeding and gingival recession. In contrast, if found statistically significant evidence between the 4th and 5th year/ during the time/ years in which the fixed single prostheses and the gingival recession were made.

KEY WORDS: restorations, periodontal and gingival health, all-ceramic, biological complications, metalceramic, single crowns.

\section{REFERENCIAS BIBLIOGRÁFICAS}

Arango, L. D.; López, A. P. \& Madrid, M. Estudio descriptivo del estado de salud gingival de un área tratada con prótesis parcial fija en pacientes atendidos en el CES de sabaneta. Revista CES Odontología, 8:136-9, 1995.

Bader, J.; Rozier, R. G. \& McFall, W. T. Jr. The effect of crown receipt on measures of gingival status. $J$. Dent. Res., 70(10):1386-9, 1991.
Castellani, D. Atlas-texto de prótesis fija: la preparación de pilares para coronas de metal-cerámica. Editorial Barcelona, España, Espaxs, 1996. Cap. $1,4$.

Dautovic-Kazazaic, L.; Redzepagic, S.; Ajanovic, M.; Gavranovic, A. \& Strujic, S. Periodontal Evaluation of Patients with Ceramic Fused-to-Metal and Acrylate Fused-to-Metal Crowns over a Period of 
1 to 5 Years. Acta Stomatol. Croat., 44(1):34-46, 2010.

Donovan, T. E. \& Cho, G. C. Soft tissue management with metal-ceramic and all-ceramic restorations. J. Calif. Dent. Assoc., 26(2):107-12, 1998.

Goodacre, C. J.; Bernal, G.; Rungcharassaeng, K. \& Kan, J. Y. Clinical complications in fixed prosthodontics. J. Prosthet. Dent., 90(1):31-41, 2003.

Güncü, G. N.; Tözüm, T. F. \& Caglayan, F. Effects of endogenous sex hormones on the periodontium-review of literature. Aust. Dent. J., 50(3):138-45, 2005.

Linares, S.; Morales, E. \& Bravo, F. Incidencia de recesiones gingivales en la clínica odontológica de la UNMSM en el año 2008. Actual. Odontol. Salud (Lima), 6(2) ,2009. Disponible en: http:// www.actualidado dontologica.com/0908/ articulo3.shtml

Maetahara, D. Prevalencia, extensión y severidad de recesiones gingivales en pacientes del hospital central de la fuerza aérea del Perú. Tesis para optar al título profesional de cirujano dentista, Universidad Nacional Mayor de San Marcos, Lima, Perú, 2006.

Mealey, B. L. \& Moritz, A. J. Hormonal influences: effects of diabetes mellitus and endogenous female sex steroid hormones on the periodontium. Periodontol. 2000, 32:59-81, 2003.

Näpänkangas, R. \& Raustia, A. Twenty-year follow-up of metal-ceramic single crowns: a retrospective study. Int. J. Prosthodont., 21(4):307-11, 2008.

Näpänkangas, R.; Salonen, M. A. \& Raustia, A. M. A 10-year follow-up study of fixed metal ceramic prosthodontics. J. Oral Rehabil., 24(10):713-7, 1997.

Newman, M.; Takei, H.; Klokkevold, P. \& Carranza, F. Carranza's clinical periodontology. Capítulos 4, 5, $7,9,35,50$ y $72.10^{\text {a }}$ ed. México DF, McGraw-Hill Interamericana, 2010.

Padbury, A. Jr.; Eber, R. \& Wang, H. L. Interactions between the gingiva and the margin of restorations. J. Clin. Periodontol., 30(5):379-85, 2003.
Sailer, I.; Pjetursson, B. E.; Zwahlen, M. \& Hämmerle, C. H. A systematic review of the survival and complication rates of all-ceramic and metal-ceramic reconstructions after an observation period of at least 3 years. Part II: Fixed dental prostheses. Clin. Oral Implants Res., 18 (Supp/ 3):86-96, 2007.

Shillingburg, H.; Hobo, S.; Whitsett, L.; Jacobi, R. \& Brackett, S. Fundamentos esenciales en prótesis fija. $3^{\mathrm{a}}$ ed. Barcelona, España, Quintessence, 2000. Cap. 1,6 y 9 .

Dirección para correspondencia:

Luis Bustos Medina

Centro de Excelencia CIGES

Dpto. Salud Pública

Facultad de Medicina

Universidad de La Frontera

Temuco

CHILE

Email: Ibustos@ufro.cl

Recibido : 27-04-2012

Aceptado: 04-06-2012 